\title{
Short-term memory using a visual shadowing procedure ${ }^{1,2}$
}

DAVID LaBERGE AND STEPHEN WINOKUR UNIVERSITY OF MINNESOTA

\section{Abstract}

Lists were constructed with digits serving as buffers and consonant items as targets, the targets being placed at various intervals from the end of the lists. All items were colored either red or black and Ss called out the color of the characters at a fast rate. At the end of each list Ss attempted to recall the consonant target item. Short-term memory functions were obtained which yielded a high recall rate at the zero retention interval and relatively low recall rate after a $6 \mathrm{sec}$. retention interval.

\section{Problem}

There are at least two factors which seem to interfere with current attempts to obtain clear representation of the short-term memory function. One factor is uncontrolled rehearsal, which attenuates the decrease in recall as a function of retention interval. A second factor emerges when one tries to minimize rehearsals by using brief exposures and intensive shadowing techniques (Moray, 1959; Peterson \& Kroener, 1964). In such cases the $\mathrm{S}$ often fails to learn or even to perceive the item, with the result that recall is far below $100 \%$ at the zero retention interval and the rest of the retention curve is shifted downward correspondingly.

The present study attempted to minimize rehearsal while assuring the occurrence of the appropriate perceptual act by adapting to the visual mode a shadowing procedure which had previously been used with the auditory mode (Cherry, 1953; Broadbent, 1958; Moray, 1959; Peterson \& Kroener, 1964).

\section{Method}

Experiment I. The 24 Ss were male and female volunteers from the introductory psychology course. A Lafayette Memory Drum presented 36 lists, each of which contained one target item (made up of one, two, or three consonants) plus 14 randomly selected digits from 1-9, omitting runs greater than two of the same digit. The item and digits were colored red or black, and the $\mathrm{S}$ was required to call out (i.e., shadow) the colors of the target and buffers and to recall the consonant item at the end of the list. The triple consonant items were selected from the Underwood \& Schulz (1960) retabulation of the Witmer norms, and were of $8 \%$ association value. The double consonant items were selected from the same source and had total frequencies of 10 or less. The single consonant items were randomly selected from the population of consonants, omitting $R$ and $B$. Digit and consonant items were colored red or black randomly, omitting runs greater than two of the same color. Three horizontal warning lines preceded each list.
Target items were placed in the lists at intervals of $0,2,6$, and 12 spaces from the end. Each consonant condition was given in a block of 12 lists, three lists for each retention interval, with intervals randomized within the blocks. The six possible orders of the three conditions were counterbalanced within each of the two separate sets of lists, and all possible orders of lists within conditions were randomized across Ss.

The presentation rate was $1 / 2 \mathrm{sec}$. per item, and consecutive lists were separated by 4 sec. The Ss were instructed to call out each color as it appeared and to attempt to recall the consonant item when the word "item" appeared at the end of each list. Prior to attempting the task the Ss were given shadowing practice on a tape containing 80 colored digits, and they met a criterion of 60 consecutive errorless trials before proceeding to the 15-item lists. Four Ss were eliminated for extended shadowing errors.

Experiment II. The $12 \mathrm{Ss}$ were presented the practice tape and lists of Experiment I, but were instructed to call out digits and the target item, without verbalizing colors. The procedure was in all other respects similar to that of Experiment $I$.

Experiment III. The 12 Ss were instructed not to call out anything in the lists until cued for recall at the end. The procedures were similar to that of Experiment I, with the omission of the practice tape.

\section{Results}

Experiment I. Shown in Fig. 1 are the effects of retention interval length upon recall for the three complexity levels of the target item, using data from lists which included no color shadowing errors. The amount of shadowing errors was approximately 20\% across all retention intervals and item conditions.

An analysis of the differences between the three item conditions at the zero retention interval yielded a p> .10 by an F-test, following an arc-sin transformation of the scores. The data were pooled for the remaining retention intervals and an F-test of the differences between the item conditions yielded a $\mathrm{p}<.001$. A simple test of slope of the memory functions was made by subtracting the $6 \mathrm{sec}$. scores from the $0 \mathrm{sec}$. scores for each S. Both a t-test and a sign-test of the differences yielded a $\mathrm{p}<.01$ for each item condition.

The recall curves for the components of the triple and double consonant items were also examined. The recall of the first consonant of a CCC was at $100 \%$ at the zero-retention interval and fell approximately linearly to $20 \%$ at the $6 \mathrm{sec}$. interval. All other single or double combinations of $\mathrm{CCC}$ items showed a recall curve which did not differ appreciably from the CCC curve of Fig. 1. The recall curve of the first or second 


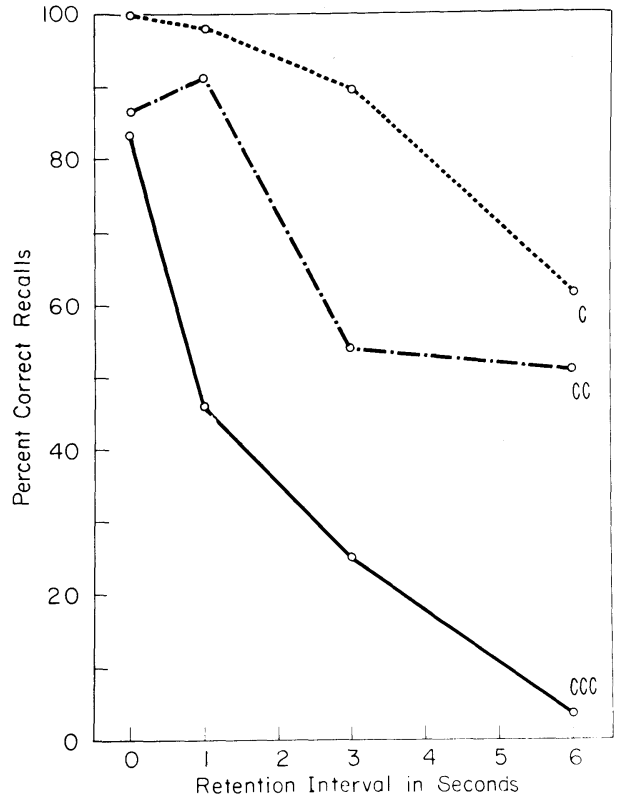

Fig. 1. Experiment I. Percent correct recalls as a function of retention interval using visual shadowing.

consonant of the CC items did not differ appreciably from the curve for both $\mathrm{CC}$ items shown in Fig. 1.

Learning effects during the course of the experiment were examined by comparing the data of the eight Ss who received an item condition first with the data of the eight Ss who received that same condition last. There appeared a slight but small trend toward better retention for the Ss who received a particular condition last.

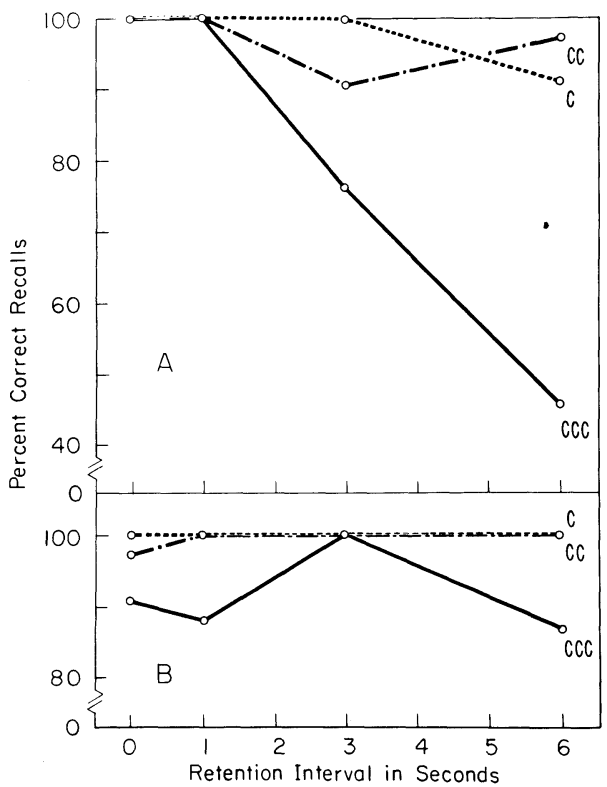

Fig. 2. Percent correct recalls as a function of retention interval. A. Experiment II: Verbalization of digits and item. B. Experiment III: No verbalizations of either digits or item.
Experiment II. The recall curve of Experiment II shown in Fig. 2 (A) is based on data from lists which yielded no shadowing errors. The only condition which shows any real retention loss is that of the CCC trigram but the amount of the loss is only about half that obtained with the procedure of Experiment I.

Experiment III. The retention curves of this experiment, shown in Fig. 2 (B), apparently show no loss over the $6 \mathrm{sec}$. interval under this experimental procedure.

\section{Diseussion}

An evaluation of the relative degree to which rehearsal was controlled by the present shadowing technique may be obtained from a comparison of the slopes of the retention curves in Fig. 1 and 2. A horizontal recall curve suggests that Ss were more likely to rehearse the items and hence prevent retention losses. However, in the absence of an independent measure of the absolute number of rehearsals performed on an item by each $\mathrm{S}$, one has no way of knowing how closely the present technique approaches the production of exactly one perceptual-learning act per trial.

Evidence that the target item was almost always perceived during the shadowing task is given by the fact that recall at the zero interval for all conditions was near $100 \%$, and that recall of the single consonant condition as well as the first letter of the threeconsonant items were precisely $100 \%$ 。 It is intuitively plausible that at the zero interval the F-test of the differences between recall scores is not sufficiently powerful to detect differences, so that an increase in the number of consonants in the target item actually decreases somewhat the probability that the item will be correctly reproduced. However, it is also likely that the immediate recall score of the longer consonant items can be raised by such procedures as increasing presentation rate and by using items of higher association value.

\section{References}

Broadbent, D. E. Perception and communication. New York: Pergamon Press, 1958.

Cherry, E. C. Some experiments on the recognition of speech with one and two ears. J. Acoust. Soc. Amer., 1953, 25, 975-979.

Moray, N. Attention in dichotic listening: Affective cues and the influence of instructions. Quart. J. exp. Psychol., 1959, 11, 56-60.

Peterson, L. R., \& Kroener, S. Dichotic stimulation and retention. J. exp. Psychol., 1964, 68, 125-130.

Underwood, B. J., \& Schulz, R. W. Meaningfulness and verbal learning. Chicago: J. B. Lippincott, 1960.

\section{Notes}

1. The research reported in this article was supported in part by grants to the University of Minnesota, Center for Research in Human Learning, from the National Science Foundation (GS 541), the National Institute of Child Health and Human Development (1-P01-HD-01136-01-HDP) and from the Graduate School of the University of Minnesota. The authors acknowledge assistance from these sources.

2. Mark Peterson and Joan Tonn assisted in the analysis of the data. 